\title{
电化学限域 $\mathrm{SiN}_{X}$ 纳米孔道检测核酸分子的研究
}

\author{
李巧, 林瑶, 应佚伦, 刘少创, 龙亿涛 ${ }^{*}$ \\ 结构可控先进功能材料及其制备教育部重点实验室; 华东理工大学化学与分子工程学院, 上海 200237 \\ *通讯作者, E-mail: ytlong@ecust.edu.cn
}

收稿日期: 2017-07-12; 接受日期: 2017-09-15; 网络版发表日期: 2017-11-21

国家自然科学基金(编号: 21505043, 21421004, 21327807)和中央高校基本科研业务费(编号: 222201718001, 222201717003, 222201714012)资 助项目

\begin{abstract}
摘要固体纳米孔道因其机械强度高、尺寸可控、易于表面修饰及集成化设计等优点被广泛应用于 DNA、RNA和蛋白质等生物分子的检测研究. 为了检测单个单链核酸分子, 本研究采用电化学刻蚀法可控 制备了单个 $\mathrm{SiN}_{X}$ 固体纳米孔道, 通过 $\mathrm{SiN}_{X}$ 固体纳米孔道限域空间效应增强了纳米孔道与短链核酸分子之间 的弱相互作用, 从而实现了核酸分子的单分子水平检测. 通过研究不同孔径 $(3.1$ 和 $8.5 \mathrm{~nm})$ 纳米孔道与核酸分 子间的弱相互作用差异, 有效区分了核酸分子在限域空间内产生的过孔和碰撞两种个体行为, 加深了对固体 纳米孔道限域空间内核酸分子电化学行为的理解.
\end{abstract}

关键词固体纳米孔道, 电化学空间限域, 核酸分子, 单分子分析

\section{1 引言}

纳米孔道技术因其操作简单、快速灵敏且无需 标记等特点而被作为一种单分子分析技术广泛应用 于 $\mathrm{DNA}^{[1 \sim 3]}$ 、多肽 ${ }^{[4 \sim 6]}$ 、蛋白质 ${ }^{[7 \sim 9]}$ 等生物分子研究. 当 单个纳米孔道被置于电解液中, 它将电解液溶液分隔 成两个独立腔室, 纳米孔道成为连接两个腔室的唯一 传质通道 ${ }^{[10]}$. 单个分子被纳米孔道捕获, 限制在纳米 孔道内腔空间内 ${ }^{[11]}$, 引起纳米孔道离子流变化, 从而 产生单个待测物分子的特征离子电流信号. 因此, 通 过纳米孔道的限域空间可以将单个生物分子的特征 结构转变成易于检测的离子流信号, 从而实现对单个 生物分子的检测 ${ }^{[12]}$.

目前, 纳米孔道被分为两类, 即生物纳米孔道和固 体纳米孔道. 生物纳米孔道多为蛋白质孔道, 在单分
子检测方面具有很高的检测灵敏度. 有别于孔径固定 的生物纳米孔道, 固体纳米孔道具有孔径可调的优势, 可应用于不同体积的单个生物分子的分析研究 ${ }^{[13]}$. 文 献报道单个DNA分子可以直链方式通过 $7 \mathrm{~nm}$ 的 $\mathrm{SiN}_{X}$ 固体纳米孔道 ${ }^{[14]}$. 利用 $8 \mathrm{~nm}$ 的石墨烯固体纳米孔道研 究 $\lambda$-DNA, 检测到 $\lambda$-DNA在溶液中的折叠和解折叠两 种构型状态 ${ }^{[15]}$. 为实现单个短链核酸分子的检测, 本研 究采用电化学刻蚀法 ${ }^{[16]}$ 可控制备了孔径小于 $10 \mathrm{~nm}$ 的 单个 $\mathrm{SiN}_{X}$ 纳米孔道, 以具有发卡结构的短链单链DNA $(\mathrm{ssDNA})^{[17]}$ 为模型检测分子, 通过限域空间效应增强 了纳米孔道与单个 $\mathrm{ssDNA}$ 分子之间的弱相互作用, 实 现了单个ssDNA分子的纳米孔道分析. 目前制备孔径 小于 $10 \mathrm{~nm}$ 固体纳米孔道的常用方法为聚焦离子束刻 蚀法 ${ }^{[18]}$ 和电子束刻蚀法 ${ }^{[19]}$, 与这些方法相比, 电化学刻 蚀法可原位、快速、可控地制备出单个固体纳米孔

引用格式: 李巧, 林瑶, 应佚伦, 刘少创, 龙䎲涛. 电化学限域 $\operatorname{SiN}_{X}$ 纳米孔道检测核酸分子的研究. 中国科学: 化学, 2017, 47: 1445-1449

Li Q, Lin Y, Ying YL, Liu SC, Long YT. Detection of single oligonucleotide with an electrochemical confined solid-state nanopore. Sci Sin Chim, 2017, 47: 1445-1449, doi: 10.1360/N032017-00110 
道. 实验结果显示, 空间限域效应较强的 $3.1 \mathrm{~nm} \mathrm{SiN} X$ 纳米孔道可有效获得单个短链ssDNA分子过孔信号, 从而实现了单个短链ssDNA分子的检测.

\section{2 实验部分}

\section{1 试剂与仪器}

三羟甲基氨基甲烷(Tris, $299.9 \%$ )、乙二胺四 乙酸(EDTA, 99.995\%)均购自阿拉丁公司 (中国); 氯化钾 $(\mathrm{KCl}, \geq 99.0 \%$ ) 购自 Sigma-Aldrich 公司 (美 国); 盐酸 $(\mathrm{HCl}, 36.0 \%$ 38.0\%购买自国药集团化学 试剂有限公司 (中国). 经 HPLC 纯化的 ssDNA (5'-

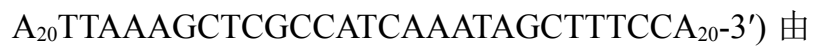
生工生物工程(上海)股份有限公司(中国)合成, 加入 Tris- $\mathrm{HCl}$ 缓冲溶液配制成浓度为 $100 \mu \mathrm{mol} / \mathrm{L}$ 的储存溶 液, 置于 $-20^{\circ} \mathrm{C}$ 保存备用. $\mathrm{SiN}_{X}$ 片购买自 Norcada公司 (加拿大). 本实验所用到的药品试剂均为分析纯, 实 验所用超纯水 $\left(18.2 \mathrm{M} \Omega \mathrm{cm}, 25^{\circ} \mathrm{C}\right)$ 由 Milli-Q系统(EMD Millipore公司, 美国)制备.

电流放大器Axopatch 200B、模数转化器Digidate $1550 \mathrm{~A}$ 、数据记录软件 Clampex 10.7 及数据处理软 件ClampFit 10.7均购自 Axon Instruments公司(美国); DELTA 320型pH计购自Mettler Toledo公司(瑞士); 等离 子体清洗仪PLASMA CLEANER PDC-32G购自Agilent Technologies公司(美国); 银丝(直径 $0.5 \mathrm{~mm}, \geq 99 \%$ )购自 Sigma-Aldrich公司(美国).

\section{2 实验方法}

实验采用 $10 \mathrm{~nm}$ 厚的 $\mathrm{SiN}_{X}$ 芯片, 其经等离子体清洗
仪处理 $30 \mathrm{~s}$ 后封装在聚四氟乙烯检测池中. 由 $\mathrm{SiN}_{X}$ 芯 片将检测池分隔成两个独立检测池, 定义为trans端和 cis端, 在检测池中分别加入电解质缓冲溶液(图1(a)). 为实现电化学刻蚀法制备单个可控固体纳米孔道, 利 用自设计 DAQ板卡通过一对Pt电极向 $\mathrm{SiN}_{X}$ 芯片两端施 加恒定直流电压, 通过LabVIEW软件记录制备过程中 $\mathrm{SiN}_{X}$ 半导体内的漏电流, 从而制备孔径可控的固体纳 米孔道. 单分子实验中产生的电流信号使用Axon 200B 膜片钳放大器和DiGiData 1550A 模数转换器进行放大 和采集. 通过 $100 \mathrm{kHz}$ 采样率, $5 \mathrm{kHz}$ 低通滤波采集电 流信号, 实验所得数据使用自编程序软件与Origin 9.2 (OriginLab Corporation, Northampton, 美国)进行统计分 析. 固体纳米孔道制备过程中所用缓冲溶液和核酸 分子实验所用缓冲溶液分别为 $\mathrm{pH} 10$ 和 $\mathrm{pH} 8$ 的 $1 \mathrm{~mol} / \mathrm{L}$ $\mathrm{KCl}(10 \mathrm{mmol} / \mathrm{L}$ Tris-HCl, 1 mmol/L EDTA $)$.

\section{3 结果与讨论}

\section{1 固体纳米孔道的制备与表征}

本研究利用本实验室已报道的电化学刻蚀法 ${ }^{[2]}$ 制 备了单个 $\mathrm{SiN}_{X}$ 固体纳米孔道, 利用LabVIEW 自编程序 软件施加恒电压 $7.5 \mathrm{~V}$, 同时记录由此在 $\mathrm{SiN}_{X}$ 纳米芯片 中产生的漏电流 $I_{\text {leakage }}$ (图1(b)), 随着施加电压的时间 增长, 漏电流在 $\mathrm{SiN}_{X}$ 纳米芯片上出现不可逆增大, 即 纳米孔道开始形成, 且当电压继续施加时纳米孔道孔 径会继续变大, 电流也随之增大, 当电流增大到预先 设定的电流阈值时, LabVIEW控制电压降至 $0 \mathrm{~V}$, 以防 止纳米孔道的进一步增大, 从而实现对纳米孔道孔径 的有效控制.
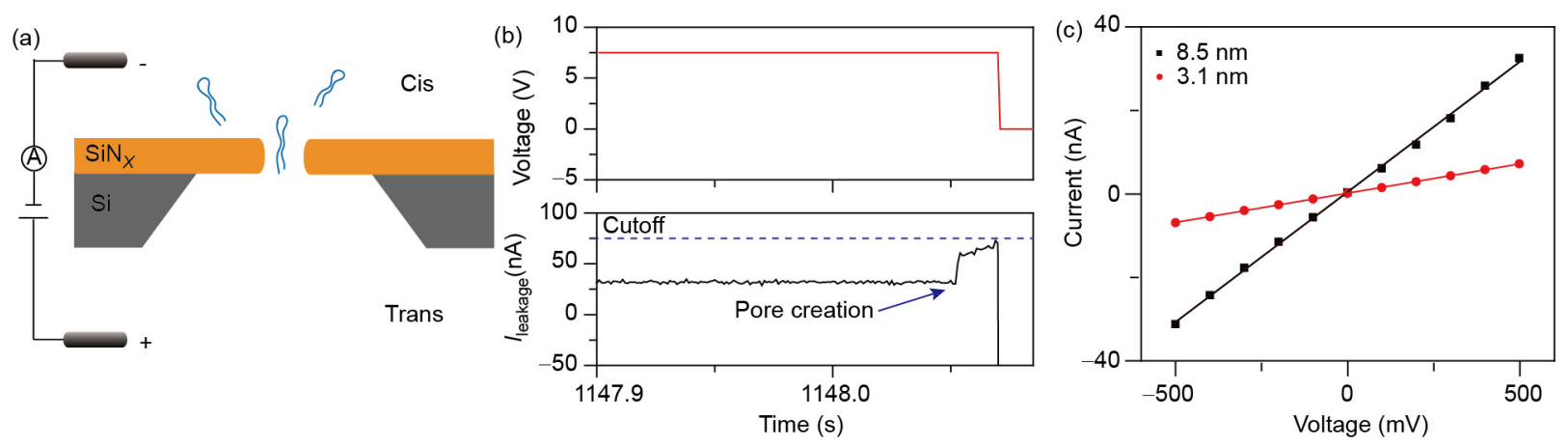

图 1 (a) 固体纳米孔道检测单个核酸分子示意图; (b) 固体纳米孔道制备过程的时间-电压(红色), 时间-电流曲线(黑色); (c) 固体纳米孔道在 $1 \mathrm{~mol} / \mathrm{L} \mathrm{KCl}$ 电解质中的 $I-V$ 曲线表征 (网络版彩图) 
图1(c)为电化学刻蚀法制备的单个 $\mathrm{SiN}_{X}$ 固体纳米 孔道在 $1 \mathrm{~mol} / \mathrm{L} \mathrm{KCl}$ 电解质缓冲溶液中的 $I-V$ 曲线, 对 $I-V$ 曲线进行线性拟合, $R^{2}$ 分别为 0.9999 (红色)和 0.9988 (黑色). 由 $I-V$ 曲线斜率计算得出单个纳米孔开孔状态 即纳米孔道内无待测物分子时的电导 $G_{0}$. 进一步, 由 电导公式 ${ }^{[20]}$ 计算纳米孔道有效直径:

$$
G_{0}=\sigma\left(\frac{4 l}{\pi d^{2}}+\frac{1}{d}\right)^{-1}
$$

其中, $\sigma$ 为电解质溶液电导率, $l$ 为 $\mathrm{SiN}_{X}$ 有效膜厚, $d$ 为开 孔状态下孔道有效直径. 文献报道 $10 \mathrm{~nm}$ 厚的 $\mathrm{SiN}_{X}$ 薄 膜, 其有效膜厚为 $3.7 \mathrm{~nm}^{[21]}$. 将 $\sigma=10.5 \mathrm{~S} / \mathrm{m}, l=3.7 \mathrm{~nm}$ 代 入式(1), 计算得出实验所用 $\mathrm{SiN}_{X}$ 固体纳米孔道有效孔 径分别为 $d_{1}=3.1 \mathrm{~nm}, d_{2}=8.5 \mathrm{~nm}$.

\section{2 固体纳米孔道检测单个核酸分子}

本研究所用模型检测分子为具有发卡结构的 ssDNA分子. 该分子可分为3部分, 即 $15-n t$ 形成的loop 环, 12-nt组成的茎部, 以及由 $\operatorname{poly}(\mathrm{dA})_{20}$ 组成的引导链.

为了检测单个ssDNA分子, 首先制备了与模型待 测分子尺寸相近的孔径为 $3.1 \mathrm{~nm}$ 的 $\mathrm{SiN}_{X}$ 纳米孔道. 将 $\operatorname{ssDNA}$ 加入含有 $1 \mathrm{~mol} / \mathrm{L} \mathrm{KCl}$ 的 $\mathrm{Cis}$ 端检测池后, 单个 ssDNA分子在电压驱动下进入纳米孔道, 产生了两种 不同阻断程度的特征电流信号(图2).

为进一步探究发卡结构ssDNA分子在 $3.1 \mathrm{~nm}$ 固体 纳米孔道单分子实验中的两种特征电流信号, 本研究 利用电化学刻蚀法可控制备了 $8.5 \mathrm{~nm}$ 孔径的固体纳米 孔道, 在相同实验条件下进行了 ssDNA单分子实验. 比 较分析不同孔径 $\mathrm{SiN}_{X}$ 固体纳米孔道限域空间内单个 ssDNA分子的实验数据, 统计结果如图3所示. 本研究 将单个阻断电流信号的电流值定义为 $I, \mathrm{SiN}_{X}$ 固体纳米 孔道的开孔电流定义为 $I_{0}$, 则单个核酸分子在固体纳米 孔道内造成的阻断电流可表示为 $I / I_{0}$, 即 $I / I_{0}$ 值越大, 阻 断程度越小. 以阻断电流比值 $I / I_{0}$ 为横坐标, 阻断时间 为纵坐标, 分别统计绘制了在 $100 \mathrm{mV}$ 电压下 $3.1 \mathrm{~nm}$ (红 色)和 $8.5 \mathrm{~nm}$ (黑色)固体纳米孔道阻断电流信号的阻 断电流比值-阻断时间散点图(图3(a)). 由散点图可以 看出, $8.5 \mathrm{~nm} \mathrm{SiN}_{X}$ 纳米孔道单分子实验中, $\mathrm{DNA}$ 产生的 阻断信号的阻断电流比值主要集中在 $I / I_{0}$ 为 $0.75 \sim 1.00$ 区域内, 表明 ssDNA与 $\mathrm{SiN}_{X}$ 纳米孔道相互作用引起的 阻断程度小, 将该区域定义为P1区, 而在孔径为 $3.1 \mathrm{~nm}$ 的纳米孔道中造成的阻断电流比值在 $I / I_{0}$ 为 $0.25 \sim 1.00$

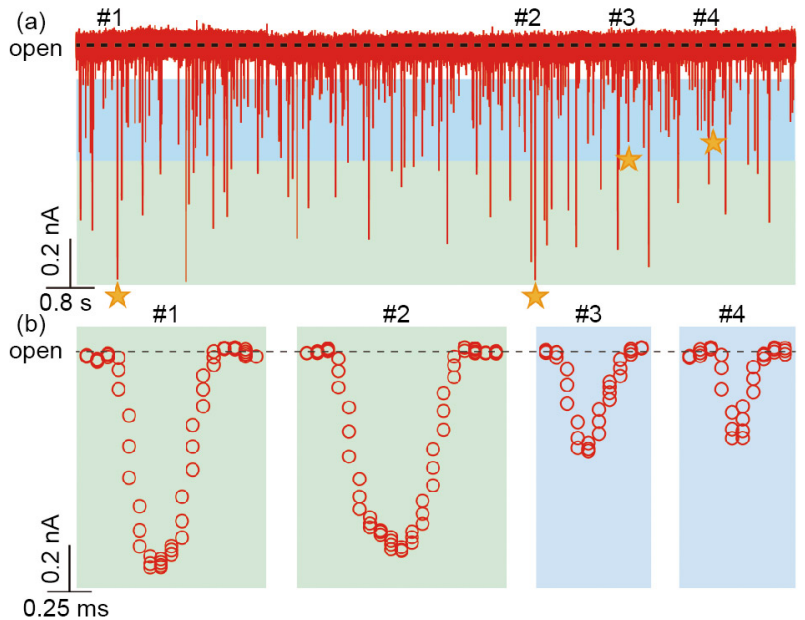

图 $23.1 \mathrm{~nm}$ 的 $\mathrm{SiN}_{X}$ 纳米孔道检测单个 $\mathrm{ssDNA}$ 分子. (a) 纳米 孔道中ssDNA产生的时间-电流原始信号; (b) 单个核酸分子 两种特征电流信号, 施加电压为 $100 \mathrm{mV}$ (网络版彩图)
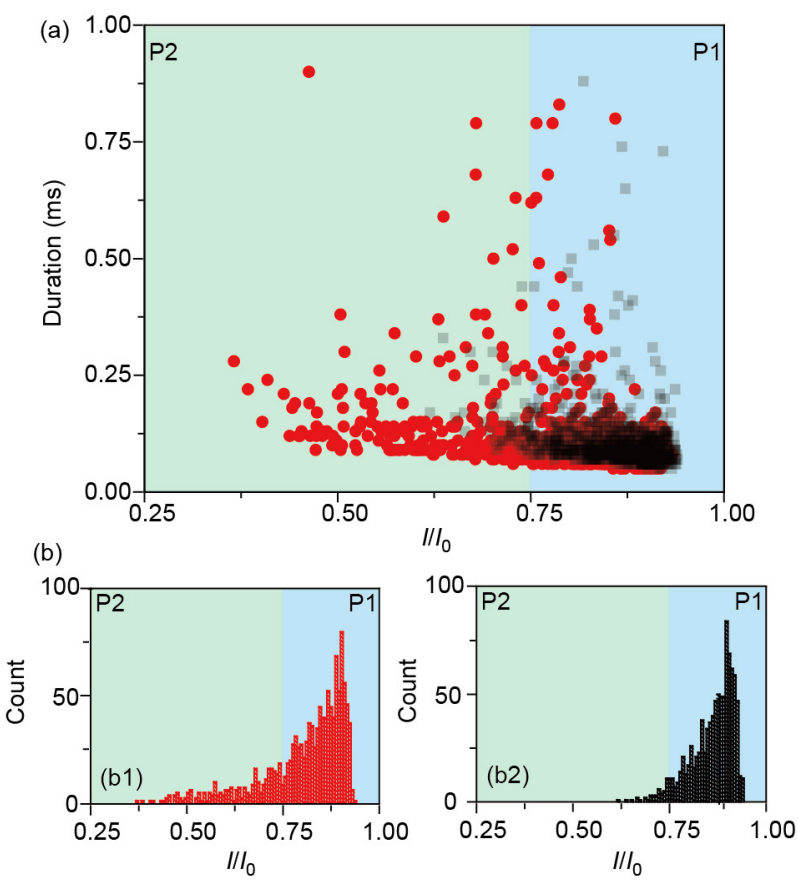

图 3 (a) 单个ssDNA分子在直径为 $3.1 \mathrm{~nm}$ (红色)和 $8.5 \mathrm{~nm}$ (黑色) $\mathrm{SiN}_{X}$ 限域纳米孔道内的阻断电流比值-阻断时间散点 图, 其中横坐标为阻断电流比值 $I / I_{0}$, 纵坐标为阻断时间; (b) $3.1 \mathrm{~nm}$ (b1)及 $8.5 \mathrm{~nm}$ (b2)阻断程度直方图. 施加电压 $100 \mathrm{mV}$ (网络版彩图)

范围内均有分布, 并将 $I / I_{0}$ 小于 0.75 的区域定义为 $\mathrm{P} 2$ 区. 比较两次不同孔径纳米孔道的单分子实验结果发 
现, 在孔径为 $3.1 \mathrm{~nm}$ 的纳米孔道实验中, 阻断信号在 $\mathrm{P} 2$ 区明显增多. 根据阻断电流比值直方图(图3(b)) 可见, $3.1 \mathrm{~nm}$ 纳米孔道的单分子实验阻断信号的阻断电流比 值在P2区分布增多明显.

文献报道, 单个ssDNA分子在限域空间内与纳米 孔道相互作用时会产生过孔和碰撞两种信号 ${ }^{[22]}$. 过孔 引起的阻断电流比值小于碰撞引起的阻断电流比值. 为计算单个短链ssDNA分子穿过固体纳米孔道的阻 断电流比值, 本文假定具有部分双链结构的单个发夹 ssDNA分子的有效直径为 $2.2 \mathrm{~nm}$, 首先由式 $(2)^{[2]}$ 计算当 单个ssDNA分子穿过纳米孔道时纳米孔道有效直径为

$$
d_{\text {with DNA }}=\sqrt{d^{2}-d_{\mathrm{DNA}}^{2}}
$$

其中, $d_{\text {with DNA }}$ 为纳米孔道内存在单个ssDNA分子时纳 米孔道的有效直径, $d_{\mathrm{DNA}}$ 为待测分子有效直径. 根 据式(3)计算出单个ssDNA穿过纳米孔道时造成的阻 断电流比值:

$$
\frac{I_{\text {with DNA }}}{I_{0}}=\frac{G_{\text {with DNA }}}{G_{0}}
$$

其中, $I_{\text {with DNA }}$ 为纳米孔道内存在单个 $\mathrm{ssDNA}$ 分子时的

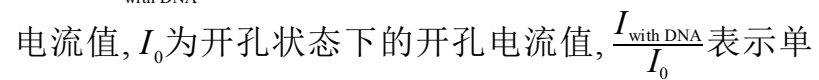
个ssDNA分子过孔引起的阻断电流比值. 计算得到
孔径为 3.1 和 $8.5 \mathrm{~nm}$ 的固体纳米孔道中存在单个待测 ssDNA分子时的 $\frac{I_{\text {with DNA }}}{I_{0}}$ 分别为 0.57 和 0.94 . 因此, 对于 $3.1 \mathrm{~nm}$ 的纳米孔道, P2范围内的阻断电流信号是由单 个ssDNA分子通过固体纳米孔道造成的; 对于 $8.5 \mathrm{~nm}$ 的纳米孔道, 单个ssDNA分子的过孔信号集中在阻断 电流比值较低的P1区. 由于 $\mathrm{ssDNA}$ 通过 $8.5 \mathrm{~nm}$ 孔引起 的阻断电流比值大, 较难区分过孔信号与碰撞信号. 因 此, $3.1 \mathrm{~nm}$ 的 $\mathrm{SiN}_{X}$ 固体纳米孔道的限域空间效应更加 显著, 从而使得单个ssDNA分子穿过限域空间产生的 阻断电流程度更大, 更利于区分ssDNA分子在限域空 间内的碰撞和过孔行为, 有效实现了单个短链sSDNA 的纳米孔道单分子水平检测.

\section{4 结论}

综上所述, 本研究应用电化学刻蚀法成功制备了 单个 $\mathrm{SiN}_{X}$ 固体纳米孔道, 实现了单个短链 $\mathrm{sSDNA}$ 分子 的纳米孔道单分子水平分析. 通过研究不同孔径(3.1 和 $8.5 \mathrm{~nm}$ )纳米孔道对单个 ssDNA分子的检测显示, 空 间限域效应较强的 $3.1 \mathrm{~nm} \mathrm{SiN}_{X}$ 纳米孔道可有效获得单 个短链ssDNA分子过孔信号, 而空间限域效应较弱的 $8.5 \mathrm{~nm}$ 纳米孔则较难区分单个短链ssDNA的纳米孔道 行为. 研究结果加深了对固体纳米孔道限域空间效应 对单个核酸分子分析机制的理解.

\section{参考文献}

Cao C, Ying YL, Hu ZL, Liao DF, Tian H, Long YT. Nat Nanotech, 2016, 11: 713-718

Lin Y, Shi X, Liu SC, Ying YL, Li Q, Gao R, Fathi F, Long YT, Tian H. Chem Commun, 2017, 53: 3539-3542

曹婵, 廖冬芳, 应佚伦, 龙亿涛. 化学学报, 2016, 74: 734-737

$\mathrm{Hu}$ YX, Ying YL, Gu Z, Cao C, Yan BY, Wang HF, Long YT. Chem Commun, 2016, 52: 5542-5545

Wang Y, Montana V, Grubišić V, Stout Jr. RF, Parpura V, Gu LQ. ACS Appl Mater Interf, 2015, 7: 184-192

Stefureac R, Long YT, Kraatz HB, Howard P, Lee JS. Biochemistry, 2006, 45: 9172-9179

Wei R, Gatterdam V, Wieneke R, Tampé R, Rant U. Nat Nanotech, 2012, 7: 257-263

Han A, Creus M, Schürmann G, Linder V, Ward TR, de Rooij NF, Staufer U. Anal Chem, 2008, 80: 4651-4658

Talaga DS, Li J. J Am Chem Soc, 2009, 131: 9287-9297

Kasianowicz JJ, Brandin E, Branton D, Deamer DW. Proc Natl Acad Sci USA, 1996, 93: 13770-13773

Long YT, Zhang MN. Sci China Ser B-Chem, 2009, 52: 731-733

Ying YL, Long YT. Sci China Chem, 2017, 60: 1187-1190

Howorka S, Siwy ZS. Nat Biotechnol, 2012, 30: 506-507

Zhang Y, Liu L, Sha J, Ni Z, Yi H, Chen Y. Nanoscale Res Lett, 2013, 8: 245

5 Merchant CA, Healy K, Wanunu M, Ray V, Peterman N, Bartel J, Fischbein MD, Venta K, Luo Z, Johnson ATC, Drndić M. Nano Lett, 2010, 10: 2915-2921

16 Kwok H, Briggs K, Tabard-Cossa V. PLoS One, 2014, 9: e92880

17 Savory N, Abe K, Sode K, Ikebukuro K. Biosens Bioelectron, 2010, 26: 1386-1391 


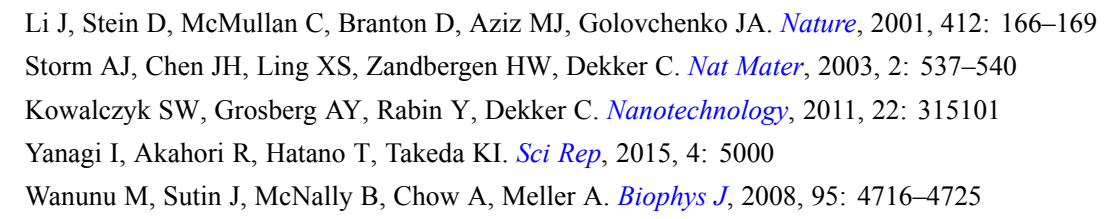

\title{
Detection of single oligonucleotide with an electrochemical confined solid-state nanopore
}

\author{
Qiao Li, Yao Lin, Yi-Lun Ying, Shao-Chuang Liu, Yi-Tao Long ${ }^{*}$ \\ Key Laboratory for Advanced Materials \& School of Chemistry and Molecular Engineering, East China University of Science and Technology, \\ Shanghai 200237, China. \\ *Corresponding author (email: ytlong@ecust.edu.cn)
}

\begin{abstract}
Solid-state nanopore has been widely used for a variety of single-molecule analysis due to its advantage of size control, high stability, adjustable surface properties, and the potential of further integration. Therefore, the insensitive studies have applied for the nanopore analysis of DNA, RNA and proteins. In order to detect an oligonucleotide at single-molecule level, here, we fabricated two $\mathrm{SiN}_{X}$ solid-state nanopores with diameter of 3.1 and $8.5 \mathrm{~nm}$ by controlled dielectric breakdown. The weak interaction between the solid-state nanopore and the oligonucleotide could be efficiently enhanced by electrochemical confined space effect of nanopore. We employed a 67-nt oligonucleotide as a model which could be electrochemically driven into a nanopore. The model ssDNA in nanopore with confined diameter of $3.1 \mathrm{~nm}$ could result in two kinds of electrochemical behaviors which assign to bumping and translocation. Our findings pave the path for a better understanding of the electrochemical behavior of the single molecule in a confined space.
\end{abstract}

Keywords: solid-state nanopore, electrochemical confined space, oligonucleotides, single-molecule analysis doi: $10.1360 / \mathrm{N} 032017-00110$ 